\title{
Descripción de la Biblioteca Digital ComPADRE Destinada a la Enseñanza y el Aprendizaje de la Física
}

\author{
Nestor L. Osorio \\ University Libraries, Reference and Research Dept., 304A, Northern Illinois University \\ DeKalb, IL 60115, EEUU (e-mail: nosorio@niu.edu)
}

Recibido Sep. 08, 2011; Aceptado Nov. 02, 2011; Versión final recibida Ene. 10, 2012

\begin{abstract}
Resumen
Se analizan los proyectos digitales para la educación científica y se hace una presentación y descripción detallada de los servicios prestados por ComPADRE, una biblioteca digital para la enseñanza y el aprendizaje de la Física. ComPADRE (sigla de Communities of Physics and Astronomy Digital Resources in Education) comenzó en 1997 cuando la Asociación Americana de Profesores de Física construyó el Centro de Recursos de Ciencias Físicas, estableciendo desde entonces activa colaboración con varias importantes sociedades científicas del mundo de la física. Del estudio y análisis se concluye que uno de los grandes atributos de ComPADRE es que los educadores pueden encontrar en un solo lugar una enorme cantidad de recursos educativos que han pasado por un cuidadoso proceso de selección.
\end{abstract}

Palabras clave: biblioteca digital, ComPADRE, enseñanza aprendizaje, educación en física

\section{Description of the Digital Library ComPADRE Intended for the Teaching and Learning of Physics}

\begin{abstract}
The use of digital resources in scientific education is discussed and a detailed description of the services of ComPADRE, a digital library created for the teaching and learning of physics is presented. ComPADRE (abbreviation of Communities of Physics and Astronomy Digital Resources in Education) started in 1997 when the American Association of Physics Teachers founded the Physical Sciences Resource Center establishing since then active collaboration with several important scientific societies of the physics world. From the study and analysis it is concluded that one of the greatest attributes of ComPADRE is that educators can find in a single place a great amount of teaching resources that have been carefully selected.
\end{abstract}

Keywords: digital libraries, ComPADRE, teaching and learning, physics education 


\section{INTRODUCCIÓN}

William Y. Arms es uno de los creadores de la conocida revista D-Lib Magazine, una publicación dedicada a la difusión de información sobre la investigación de la biblioteca digital y sus aplicaciones. Su libro Digital Libraries (Arms, 2000) presenta una perspectiva general de este campo emergente. Se recomienda su lectura especialmente para los interesados en la tecnología actual de la publicación y preservación de conocimientos académicos; este libro contiene una recopilación valiosa de información sobre las bibliotecas digitales. En este libro Arms presenta las áreas más relevantes de las bibliotecas digitales, tales como: modelos de objetos; interfaces de usuario y la interacción persona-ordenador; el descubrimiento de información; metadatos descriptivos; procesamiento de lenguaje natural; los materiales no textuales; la gestión y preservación de colecciones; organización; archivo y conservación; conversion; interoperabilidad (que incluye entre otros temas de interfaces de usuario; protocolos de red; y autenticación y seguridad); escalabilidad; fiabilidad y robustez; y los problemas económicos, sociales y legales. De hecho, el área de las bibliotecas digitales se está convirtiendo en una materia compleja, que combina temas de tecnología de la información, ciencias de la información, ciencias de la computación, y otros temas. En América Latina hay varias organizaciones y grupos de profesores implicados en el desarrollo de bibliotecas digitales. Uno de los esfuerzos más destacados es el portal SciELO < http://www.scielo.org/php/index.php?lang=es >. SciELO contiene cientos de revistas de investigación científica con texto completo proveniente de los 10 países que más contribuyen a este proyecto, al igual que muchos otros títulos de otros países.

Studart (2011) muestra la importancia de formar un equipo multidisciplinario en el marco de la creación de objetos educativos para la Física. Este equipo debe ser uno en el que estudiantes y profesores expertos en el área de conocimiento trabajen en colaboración con educadores, expertos en medios de comunicación, programadores y diseñadores web a fin de lograr un producto de alta calidad. El autor también informa sobre las actividades en un distrito escolar de Brasil donde se lleva cabo la capacitación para ayudar a los maestros a aprender las nuevas tecnologías y evaluar sus resultados. Además, Santos Miranda, Riposati Arantes, y Studart (2011) proponen el uso de objetos de simulación interactiva del proyecto Tecnología para la Educación de la Física (Physics Education Technology) (PhET) de la Universidad de Colorado, EE.UU., para la enseñanza y el aprendizaje de la Física en las instituciones educativas en Brasil.

\section{RECURSOS EDUCATIVOS DIGITALES EN LÍNEA}

Desde la creación de la web una nueva área de enseñanza se inicio basada en el diseño con el uso de tecnologias informática de recursos educativos. Inicialmente, los maestros motivados por su propria cuenta desarrollaron estos recursos, los rápidos avances de la tecnología de la información han permitido la expansión de los modelos de enseñanza y la producción más organizada de recursos de enseñanza; esto se ha logrado en parte por el interés público en la creación de nuevos métodos educativos. Las instituciones entonces comenzaron a recibir fondos de agencias gubernamentales y de organizaciones privadas para este fin.

Existe una amplia literatura sobre el uso educativo y valor de los objetos de aprendizaje que incluye artículos académicos, libros, tesis doctorales e informes; un análisis completo de este tema está fuera del alcance de este artículo. Publicaciones recientes son por ejemplo: Teaching Strategies in the Online Environment (Conceição, 2007), Web-based Education and Pedagogical Technologies: Solutions for Learning Applications (Esnault, 2008), Designing Effective Instruction (Morrison et al, 2011.), y la Revista Brasileira de Ensino de Física en 2002 le dedicó un número especial al el uso de estas tecnologías en la enseñanza de la Física.

El uso de objetos de enseñanza por ordenador y las simulaciones en las ciencias es un campo activo. Heckler et al. (2007) reporta el desarrollo y el uso de un paquete informático basado en módulos educativos para la enseñanza secundaria de la Óptica. Las opiniones obtenidas de los estudiantes calificaron altamente este recurso, cumpliendo con sus expectativas, y proporcionando un entorno para estimular su participación en el proceso de aprendizaje. Serrano y Narváez (2010) discute sobre el desarrollo y el uso del Sistema de Aprendizaje Virtual 
Interactivo (SAVIO) en la Universidad Tecnológica de Bolívar en Cartagena, Colombia. Los cambios en el tradicional proceso de enseñanza aprendizaje puede ser adoptado por otras instituciones, el autor afirma. Una cosa que hace este proyecto interesante es el uso en su totalidad de desarrollo de software libre.

Las bibliotecas digitales educativas son tambien parte de estos esfuerzos. Una biblioteca digital educativa, no sólo incluye en su contenido material de lectura, sino que, más importante todavía, incluye recursos didácticos apropiados para la enseñanza de una materia específica. Cabral Vargas (2006) explica los esfuerzos necesarios para crear materiales educativos de alta calidad. Para lograr el aprendizaje significativo a través de la utilización de materiales didácticos basados en la web, muchos elementos necesarios deben ser tomados en consideración. Un elemento muy importante de acuerdo a Cabral Vargas es la adquisición secuencial del conocimiento de los estudiantes: "la BD tiene una tarea ardua en el diseño de materiales, ya que no basta con saber lo que se desea enseñar, sino que también hay que identificar los conocimientos previos con los que cuentan los alumnos, con la finalidad de examinar, organizar y presentar los materiales del curso de tal manera que se incremente la comprensión, la retención y el aprendizaje del estudiante; apoyando al especialista que asesora dichos contenidos a desarrollar el contexto adecuado, para que el estudiante construya su conocimiento y desarrolle habilidades y valores." La creación de contenidos con buena calidad es fundamental para la utilización eficaz y práctica de estos recursos educativos.

Las simulaciones son un método muy importante para la enseñanza de conceptos de las ciencias, Adams et al. (2008) publicó los resultados de una encuesta en la que 200 estudiantes fueron entrevistados durante sus interacción con las simulaciones de unos temas de Física. Llegaron a la conclusión de que hay "pruebas abrumadoras de que las simulaciones que incorporen adecuadamente la interactividad, animación, y el contexto pueden proporcionar un ambiente de aprendizaje de gran alcance donde los estudiantes de manera productiva pueden colaborar en alcanzar a dominar el contenido de la Física. Sin embargo, encontraron que esto sólo puede lograrse después de aplicar un extenso conjunto de principios para su diseño y su presentación.

A pesar de todas las ventajas posibles, hay algunos obstáculos para el uso de bibliotecas digitales educativas que contienen materiales de enseñanza, tales como simulaciones, objetos interactivos y otros. McMartin et al. (2008) realizó una encuesta a profesores de educación superior en los Estados Unidos con el objeto de determinar su comprensión y uso de bibliotecas digitales y las barreras que limitan su uso. Ellos encontraron que los datos demográficos típicos recogidos, tales como la experiencia en la enseñanza, no era pertinente para determinar la población de usuarios. Sólo ciertos tipos de recursos digitales tienen un mayor uso. La falta de tiempo debido a las muchas obligaciones profesionales fue una fuerte barrera, así como una falta de confianza en los materiales didácticos conseguidos en el Internet.

Estas nuevas metodologías de enseñanza no siempre son bien aceptadas y con frecuencia estan sometidas a escrutinio (Tobias y Duffy, 2009), "existen muchos estudios que indican limitaciones a las prácticas de aprendizaje por descubrimiento." Existe una necesidad por más investigación sólida en este campo. El trabajo realizado por Baki y Çakıroğlu (2010) y por Kay y Knaack (2007) representan buenas aportaciones. En el primer estudio el uso de objetos de aprendizaje en los cursos de Matemáticas en secundaria fue examinado. Los datos cualitativos y cuantitativos obtenidos de los profesores y los estudiantes indican "que los estudiantes eran fácilmente capaces de seguir las instrucciones de las actividades de enlace y la práctica dentro de LOs (Learning objects)." Además, los estudiantes también suelen participar en el proceso de aprendizaje. Los autores concluyen que "el uso de LOs puede ser un instrumento de aprendizaje eficaz en los cursos de Matemáticas en la secundaria." Kay y Knaack evaluaron el uso de objetos de aprendizaje para las ciencias en la escuela secundaria para las áreas de la Biología, la Química y la Física. En este caso la mayoría de los estudiantes informarón que se benefician de la utilización de objetos de aprendizaje, los maestros también están de acuerdo en su valor y tenían previsto continuar utilizando estos recursos. 
Después de una exhaustiva búsqueda en múltiples bases de datos, se puede concluir que la literatura profesional sobre ComPADRE publicada en revistas especializadas son escasas, la mayoría de los artículos son publicados en las actas de conferencias, informes elaborados por su personal técnico, y presentaciones en los talleres de enseñanza. Esta es la razón por la cual los documentos indicados en la Tabla 6 son principalmente de estos tipos. Además, una evaluación imparcial con comentarios de los usuarios sobre los servicios y colecciones de ComPADRE no se ha publicado. Este es sin duda un tema de potencial investigativo para una tesis doctoral sobre la educación de las ciencias.

\section{ComPADRE}

Uno de los proyectos de bibliotecas digitales más importantes en América del Norte relacionado con colecciones digitales para la ciencia y la tecnología ha sido la creación de la Biblioteca Digital Nacional para las Ciencias (National Science Digital Library) (NSDL). NSDL es un programa de la Fundación Nacional de Ciencias (National Science Foundation) (NSF) de los Estados Unidos. Originalmente, el órgano administrativo central de la NSDL fue el Equipo de Integración, un equipo formado por tres investigadores principales, diez jefes de proyecto, y tres enlaces de audiencia. En los últimos años, la administración de la NSDL ha sido contratada para la Corporación Universitaria de Investigación Atmosférica (University Corporation for Atmospheric Research) (UCAR), Boulder, Colorado. Esta nueva organización de la NSDL tiene dos divisiones principales: el Centro de Recursos y La Red de Servicios Técnicos. También es asistido por un Consejo de Adquisiciones. Bajo esta nueva estructura, el Centro de Recursos ofrece asistencia, establece alianzas estratégicas, evalúa los programas, y difunde información. La Red de Servicios Técnicos opera la infraestructura, presta apoyo técnico y define las prioridades para las iniciativas técnicas.

Desde su creación en 2000, la NSDL ha financiado cientos de proyectos en torno a cinco rutas principales de desarrollo: Sistema básico de integración, Colecciones, Servicios, Investigación dirigida y Senderos (Pathways). Además, otros organismos de financiación federal (por ejemplo, la Dirección de Geociencias (GEO) y la Dirección de Ciencias Matemáticas y Físicas (MPS) han proporcionado un importante co-financiación de estos proyectos (Osorio, 2006). La complejidad de la NSDL está bien demostrada por los elementos incluidos en estas rutas temas: proyectos de Sistema básicos de integración son para la coordinación y administración de las colecciones básicas y los servicios de la biblioteca. Proyectos de Colecciones son para administrar y consolidar un subconjunto de los contenidos de la biblioteca dentro de un campo académico. Proyectos de Servicios desarrollan servicios de apoyo a los usuarios y los proveedores de la colección. Proyectos de Investigación orientada investigan cómo los recursos digitales pueden tener un impacto educativo (prácticas, el comportamiento del usuario, la creación de nuevos entornos de aprendizaje, etc.) Proyectos de Senderos proporcionan las funciones de administración de los contenidos educativos y/o los servicios que necesitan una amplia comunidad de estudiantes (Zia, 2001). NSDL tiene incorporada actualmente dieciocho Senderos de campo académicos, Tabla 1.

La palabra 'compadre' en español con frecuencia se utiliza para referirse a un buen amigo, alguien en quien confiar. Los creadores de este portal especializado más probable es que hubíaran elegido este nombre para indicar el buen amigo - en el que uno puede confiar - para la comunidad de la enseñanza de la Física. En realidad, y por pura coincidencia, el nombre proviene del título original en Inglés del portal: Communities of Physics and Astronomy Digital Resources in Education (ComPADRE) (Deustua, 2004). El origen de ComPADRE comenzó en 1997, cuando la Asociación Americana de Profesores de Física (American Association of Physics Teachers) (AAPT) construyó el Centro de Recursos de Ciencias Físicas (Physical Sciences Resource Center) (PSRC) <http://psrc.aapt.org >. En 2003, PSRC comenzó a formar parte de la NSDL, y en el 2005, ComPADRE fue financiado por primera vez y comenzó su papel como uno de los Senderos temáticos de la NSDL. ComPADRE también ha establecido colaboración con la Sociedad Astronómica Americana (American Astronomical Society) (AAS), el El Instituto Americano de Física (American Institute of Physics) (AIP), y la Sociedad Americana de Física (American Physical Society) (APS). Este ha sido financiado por la NSF a través de la NSDL. 
Tabla 1: Los Senderos de ComPADRE

\begin{tabular}{|c|c|}
\hline $\begin{array}{l}\text { AMSER: Sendero de Matemáticas Aplicada y } \\
\text { Ciencias }\end{array}$ & AMSER: Applied Math and Science Pathway \\
\hline BEN: Sendero de Ciencias Biológicas & BEN: Biological Sciences Pathway \\
\hline ChemEd DL: Sendero de Educación de la Química & ChemEd DL: Chemistry Education Pathway \\
\hline $\begin{array}{l}\text { CLEAN: Sendero de la Red de Sensibilización para } \\
\text { el Conocimiento Informativo del Clima y la Energía }\end{array}$ & $\begin{array}{l}\text { CLEAN: Climate Literacy and Energy Awareness } \\
\text { Network Pathway }\end{array}$ \\
\hline ComPADRE: Sendero de Física y Astronomía & ComPADRE: Physics and Astronomy Pathway \\
\hline $\begin{array}{l}\text { Sendero de Recursos para la Educación de la } \\
\text { Ciencias de la Computación }\end{array}$ & $\begin{array}{l}\text { CSERD: Computational Science Education } \\
\text { Resources Pathway }\end{array}$ \\
\hline $\begin{array}{l}\text { DLESE: Biblioteca Digital de la Educación de la } \\
\text { Tierra }\end{array}$ & DLESE: Digital Library for Earth System Education \\
\hline $\begin{array}{l}\text { Sendero de Ingeniería: Recursos para la } \\
\text { Enseñanza de la Ingeniería }\end{array}$ & $\begin{array}{l}\text { Engineering Pathway: Engineering Education } \\
\text { Resources }\end{array}$ \\
\hline $\begin{array}{l}\text { Ensemble: Sendero de las Ciencias de la } \\
\text { Computación }\end{array}$ & Ensemble: Computing Pathway \\
\hline $\begin{array}{l}\text { ICPALMS: Sendero para la Instrucción Basada en } \\
\text { Normas }\end{array}$ & $\begin{array}{l}\text { ICPALMS: Pathway for Standards-based } \\
\text { Instruction }\end{array}$ \\
\hline $\begin{array}{l}\text { MatDL: Sendero de la Biblioteca Digital para } \\
\text { Ciencias de los Materiales }\end{array}$ & MatDL: Materials Digital Library Pathway \\
\hline MathDL: Sendero de Matemáticas & MathDL: Mathematics Pathway \\
\hline $\begin{array}{l}\text { Mathlanding: Sendero para Matemáticas } \\
\text { Elementales }\end{array}$ & Mathlanding: Elementary Mathematics Pathway \\
\hline $\begin{array}{l}\text { Portal de Escuela Medial: Matemáticas y Ciencias } \\
\text { (MSP2) }\end{array}$ & $\begin{array}{l}\text { Middle School Portal: Math and Science Pathways } \\
\text { (MSP2) }\end{array}$ \\
\hline $\begin{array}{l}\text { SMILE: Sendero para el aprendizaje informal de } \\
\text { Educadores de Ciencias y Matemáticas }\end{array}$ & $\begin{array}{l}\text { SMILE: Science and Math Informal Learning } \\
\text { Educators Pathway }\end{array}$ \\
\hline $\begin{array}{l}\text { Enseñar acerca de la Tierra: Portal del SERC para } \\
\text { Educadores Ciencias de la Tierra }\end{array}$ & $\begin{array}{l}\text { Teach the Earth: the SERC Portal for Geoscience } \\
\text { Educators }\end{array}$ \\
\hline $\begin{array}{l}\text { Dominio de los Maestros: Sendero de Recursos } \\
\text { para Medios Digitales }\end{array}$ & $\begin{array}{l}\text { Teachers' Domain: Digital Media Resources } \\
\text { Pathway }\end{array}$ \\
\hline $\begin{array}{l}\text { TeachingWithData.org: Conocimiento Informativo } \\
\text { Cuantitativo de las Ciencias Sociales. }\end{array}$ & $\begin{array}{l}\text { TeachingWithData.org: Pathway to Quantitative } \\
\text { Literacy in the Social Sciences. }\end{array}$ \\
\hline
\end{tabular}

Bruse Mason, su investigador principal, ha descrito ComPADRE como un portal que "reúne recursos de enseñanza, actividades de aprendizaje, y la investigación en educación. El portal organiza estos recursos a través de la labor de los editores y bibliotecarios, y los presenta al mundo. Los usuarios de la biblioteca pueden encontrar materiales en este portal, pero también pueden sugerir recursos, creación de colecciones privadas o públicas, y compartir sus conocimientos a través de discusiones, comentarios y críticas." (Mason, 2007). Las siguientes secciones de este documento se concentran en describir los elementos más importantes de este portal. 


\section{ORGANIZACIÓN Y SERVICIOS DE COMPADRE}

Desde que ComPADRE comenzó a ser un Sendero de la Biblioteca Digital Nacional para las Ciencias, el acceso a ComPADRE se puede obtener desde el enlace de la NSDL por medio de su lista de Senderos temáticos <http://nsdl.org/>. Su URL directo es: http://www.compadre.org/, si usted es suscriptor de ComPADRE es recomendable tener este enlace en la lista de favorites de su navegador web. La primera página de este portal incluye varias etiquetas (tags) que guían a los usuarios a sus colecciones y servicios, estas etiquetas son: Explora las colecciones (Explore the Collections), Eventos (Events), Socios (Partners), Gente (People), Colaborar (Collaborate), Servicios a la Comunidad (Community Services), Contacto (Contact Us), y Acerca de ComPADRE (About ComPADRE). Figura 1.

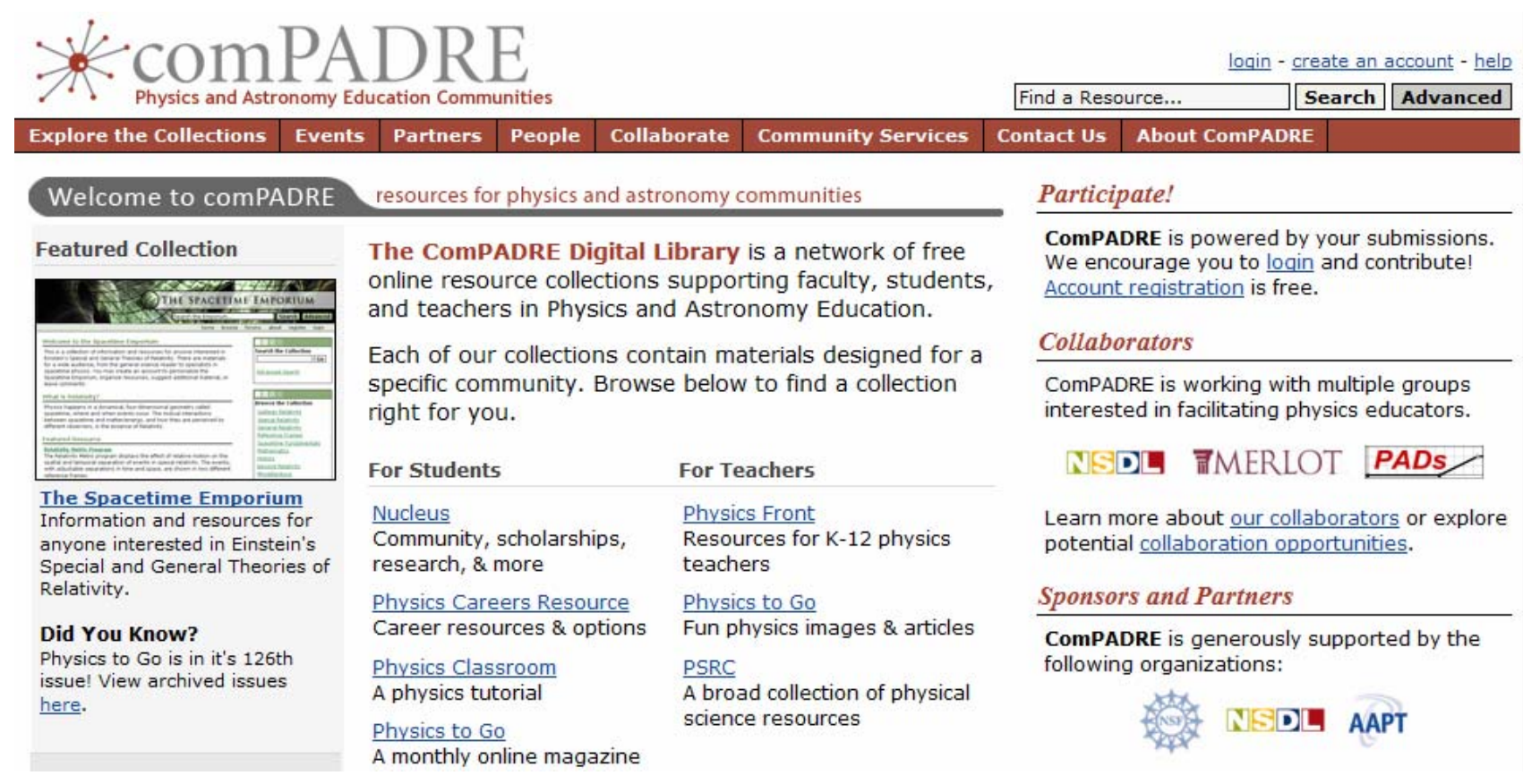

Fig. 1: La primera página de ComPADRE

Estas etiquetas también representan las principales secciones del portal. ComPADRE esta parcelado en cinco categorías principales de información:

1. Herramientas de Contenido: Explora las colecciones y un motor de Búsqueda Avanzada.

2. Herramientas de la Comunidad: Eventos, Servicios a la Comunidad y Contacto.

3. Información acerca de ComPADRE: Acerca de ComPADRE, Sociosy Gentes. También hay páginas para: Ser un colaborador y de Tecnología.

4. La sección de documentación incluye: Portal de Ayuda, Condiciones de Uso, Política de Uso Aceptable, Preguntas Frecuentes y Política de privacidad.

\section{Su cuenta.}

La sistemática de archivo de los objetos incluidos en ComPADRE es descubierto al examinar las opciones de búsqueda disponibles. Todo el portal se puede explorar usando el motor simple de búsqueda o su versión avanzada. Una exploración utilizando el motor de Búsqueda Avanzada permite tres niveles de especificidad en la búsqueda de los temas: Asuntos generales, Temas específicos, y Detalle de los tópicos, tales como: Astronomía, la Cosmología, y la Materia oscura. También es posible seleccionar el tipo de recursos necesarios, lo que incluye: Recursos didácticos; Actividades de aprendizaje; los Recursos para los estudiantes; Materiales A/V; Comunidades y eventos; Herramientas y bases de datos; Materiales de referencia; y Colecciones. Una selección más refinada se puede hacer al definir el Nivel objetivo: Educación no formal 
(pública); Escuela Primaria (K-4), Escuela Intermedia (5-8), Escuela Secundaria (9-12); Cursos iniciales de pregrado (LLUG); Cursos superiores de pregrado (ULUG); Graduado/Profesional (GRAD); y Desarrollo Profesional. Por último, una sección de Grupo de Destino también está disponible para: Estudiante; Educador; Investigador, Profesional; Administrador; Público en general; y Padre/Tutor. Por lo tanto, las búsquedas realizadas para la Astronomía, la Cosmología y la Materia oscura pueden ser limitadas a los Recursos didácticos; para Cursos iniciales de pregrado (LLUG); y para Educadores. Esta búsqueda - que es muy específica - produjo el 25 de Julio de 2011 un solo resultado. También hay una opción para los recursos sin costo o para comprar. Vea la opción de Búsqueda Avanzada en la Figura 2.

Advanced Search

Search Terms:

Search the Compadre Portal Collection

o. Search all comPADRE Collections

Limit Returned Materials:

General Subject:

Specific Subject:

Subject Detail:

Target Level:

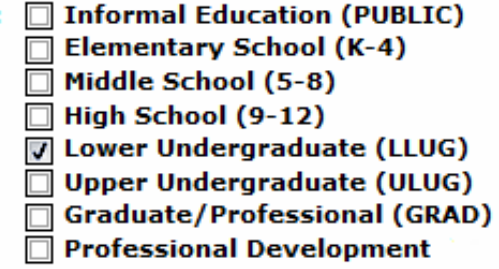

Cost: No preference -

Resource Type: Teaching Resources

Target Role:

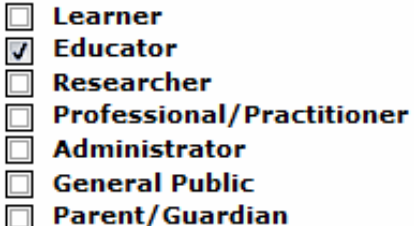

Search!

Fig. 2: Motor de Búsqueda Avanzada de ComPADRE

En la primera página, el enlace "crear una cuenta" también está disponible (Figura 1). No hay ningún costo para convertirse en un miembro de ComPADRE. Un usuario, como miembro, puede utilizar completamente todas las herramientas que ofrece este portal, participar en foros, recomendar recursos, y es propietario de un armario para guardar los materiales. Un nombre válido y la dirección de correo electrónico es todo lo que se necesita para convertirse en miembro.

En términos de organización administrativa, ComPADRE está dirigido por Bruce Mason (AAPT) como investigador principal. Los Co-Investigadores Principales son: Susana Deustua (AAS), Jack Hehn (AIP/MSF), Warren Hein (AAPT), Ted Hodapp (APS), y Gary White (SPS Director). También cuenta con tres posiciones como miembros del personal: un Líder técnico, un Editor colaborador y un Diseñador web. Cada colección tiene un Editor de la colección y, finalmente, hay tres evaluadores.

\section{LAS COLECCIONES DE COMPADRE}

El mayor valor de ComPADRE como un portal educativo se concentra en las dieciocho colecciones contenidas en este sitio. Estas colecciones están orientadas hacia cuatro audiencias diferentes: Recursos para Maestros, Colecciones Generales para los Docentes, Colecciones Específicas para Cursos y para los Docentes y los Recursos para los Estudiantes. En esta sección, se presentan los cuatro segmentos diferentes de esta biblioteca digital. Las Tablas 2 a 5 contienen una breve descripción de las colecciones incluidas en cada una de estas cuatro orientaciones. Algunas colecciones están indicadas en más de una categoría. 
Tabla 2: Recursos para Maestros

\section{El Frente de Física (The Physics Front)}

Incluye recursos didácticos K-12 para maestros de la Física. La sección sobre Tipo de Curso y Tema incluye: K-8 Ciencias Físicas; Primero Física, Física Conceptual; Basada en Algebra; AP-Cálculo. También se puede usar Navegar la Colección, Compartir Carpetas, hay una página para los Maestros Nuevos, y otra para los Normas educativas, así como oportunidades de participar.

http://www.compadre.org/precollege/

\section{Adoptar-a-un-Físico (Adopt-a-Physicist)}

Con el apoyo de la Sigma Pi Sigma $(\Sigma \Pi \Sigma)$, la sociedad de estudiantes de honor de Física, este servicio establece comunicación entre los estudiantes de secundaria de Física con los universitarios de post-grado de Física. http://www.adoptaphysicist.org/

\section{El Aula de Física (The Physics Classroom)}

Este sitio contiene tutoriales en línea para cursos de Física secundaria y está escrito para estos estudiantes. Estos recursos proporcionan una mejor comprensión de los conceptos de la Física. http://www.physicsclassroom.com/

\section{Física para LLevar (Physics to Go)}

Esta es una revista en línea y una colección de sitios educativos. Hay 4 secciones publicadas regularmente: Física en su Mundo; Investigación de la Física, Física en la Casa, y Vale la Pena Ver. http://www.compadre.org/informal/

\section{El Centro de Recursos de Ciencias Físicas-PSRC (The Physical Sciences Resource Center- PSRC)}

Es un banco de datos para los profesores de Física en todos los niveles con información sobre los materiales curriculares, las demostraciones en clase, sesiones de laboratorio, la literatura de la ciencias de la educación, entre otros. http://www.compadre.org/psrc/

\section{Tabla 3: Colecciones Generales para los Docentes}

\section{El Centro de Recursos de Ciencias Físicas-PSRC (The Physical Sciences Resource Center- PSRC)}

Es un banco de datos para los profesores de física en todos los niveles con información sobre los materiales curriculares, las demostraciones en clase, sesiones de laboratorio, la literatura de la ciencias de la educación, entre otros. http://www.compadre.org/psrc/

\section{Física de Código Abierto (Open Source Physics)}

Esta colección incluye: recursos computacionales para la enseñanza, simulaciones, modelado EJS, paquetes de currículos, recursos para programación y otras herramientas. http://www.compadre.org/osp/

\section{PER-Central (Physics Education Research Central)}

El Centro de Investigaciónes para la Enseñanza de la Física contiene información acerca de conferencias, programas educativos, apoyo curricular, una lista de tesis y disertaciones, y una base de datos acerca de la literatura de la educación de la Física. http://www.compadre.org/per/

\section{Coalición del Profesor para la Educación de la Física (The Physics Teacher Educación Coalición)}

Este es el sitio web de la PTEC, una organización que tiene como miembros a 235 instituciónes dedicadas a mejorar la educacion de la Física y la preparación del profesorado de Ciencias Físicas.

http://www.compadre.org/ptec/

\section{UComp}

Esta es una colección y comunidad de Física computacional con recursos en quince grandes áreas como Astronomía, Física Moderna, Física Cuántica y Óptica. http://www.compadre.org/ucomp/ 


\section{Tabla 4: Colecciones Específicas para Cursos y para los Docentes}

\section{Laboratorios Avanzados (Advanced Labs)}

Es una colección que tiene una amplia cobertura para profesores universitarios que enseñan los laboratorios de nivel superior de pregrado. Incluye manuales de laboratorio, programas de computadora (software) otra información relevante y foros de discusión. http://www.compadre.org/advlabs/

\section{La fuente de la Física (The Physics Source)}

La fuente de la Física es una colección que proporciona los recursos para cursos de Introducción a la Física a nivel universitario. Incluye materiales para los cursos de Mecánica, Física Térmica y Fluidos, la Electricidad y el Magnetismo, las Ondas y la Óptica y Física Moderna. http://www.compadre.org/introphys/

\section{Física Estadística y Térmica (Statistical and Thermal Physics)}

Este es un lugar donde los instructores pueden encontrar un conjunto de simulaciones de Física Estadística y Térmica, e incluso tienen acceso en línea a los materiales complementarios de un reconocido libro de Física Térmica. http://www.compadre.org/stp/

\section{Centro de Astronomía (Astronomy Center)}

Esta colección ofrece recursos para los cursos de Introducción a la Astronomía. Incluye las siguientes áreas: Astronomía, Educación, Cosmología, Fundamentos, Astronomía Histórica, la Vía Láctea, la Exploración Espacial, El Sol, Tiempo cósmico y la distancia, Exoplanetas, Galaxias, Instrumentación, Sistema solar, y las Estrellas. http://www.compadre.org/astronomy/

\section{El Intercambio del Cuántico (The Quantum Exchange)}

Colección de recursos para los instructores de los cursos de Física Cuántica. Los temas incluidos son: técnicas de aproximación, sistemas consolidados del estado, entrelazamiento e información cuántica, fundamentos y teoría de la medición, sistemas de multi-partículas, probabilidad, ondas, Interferencias, dispersión y sistemas de continuidad del estado, y rotación y sistemas de dimensión finitos.

http://www.compadre.org/quantum/

\section{Emporium Espacio-Tiempo (Spacetime Emporium)}

En esta colección, los profesores encontrarán una extensa fuente de información y recursos acerca de las Teorías Especial y General de la Relatividad de Einstein. http://www.compadre.org/relativity/

\section{Tabla 5: Recursos para los Estudiates}

\section{El Núcleo (The Nucleus)}

Un servicio para prestar apoyo a los estudiantes de pregrado de Física y Astronomía. Cubre los clubes de estudiantes, programas de investigación de verano, las oportunidades de becas, los grupos de discusión, los concursos y otras actividades. http://www.compadre.org/student/

\section{El Aula de Física (The Physics Classroom)}

Este sitio contiene tutoriales en línea para cursos de Física secundaria y está escrito para estos estudiantes. Estos recursos proporcionan una mejor comprensión de los conceptos de la Física.

http://www.physicsclassroom.com/

\section{Física para LLevar (Physics to Go)}

Esta es una revista en línea y una colección de sitios educativos. Hay 4 secciones publicadas regularmente: Física en su mundo; Investigación de la Física, Física en la casa y Vale la pena ver.

http://www.compadre.org/informal/

\section{Adoptar-a-un-Físico (Adopt-a-Physicist)}

Con el apoyo de la Sigma Pi Sigma $(\Sigma \Pi \Sigma)$, la sociedad de estudiantes de honor de Física, este servicio establece comunicación entre los estudiantes de secundaria de Física con los universitarios de post-grado de física. http://www.adoptaphysicist.org/

\section{Guías para las Carreras en Física (The Physics Careers Resource)}

Este servicio proporciona información para estudiantes, educadores y padres de familia. Muestra datos acerca de las oportunidades de empleo, los perfiles de algunos físicos, lista de los empleadores para físicos, instituciones educativas, y una visión general de los campos de la Física. http://www.compadre.org/careers/ 
Tabla 5. (continuación)

\section{El Centro de Recursos de Ciencias Físicas-PSRC (The Physical Sciences Resource Center- PSRC)}

Es un banco de datos para los profesores de Física en todos los niveles con información sobre los materiales curriculares, las demostraciones en clase, sesiones de laboratorio, la literatura de la ciencias de la educación, entre otros. http://www.compadre.org/psrc/

Tabla 6: Selección de trabajos sobre ComPADRE publicados en revistas profesionales y presentados en conferencias y reuniones

\section{Física de Código Abierto}

Belloni, M., The Evolution of the Open Source Physics ComPADRE Collection, AAPT 2010 Summer Meeting, Portland, Or. USA (2010).

Belloni, M. W. Christian y D. Brown, Open Source Physics Curricular Material for Quantum Mechanics, Computing in Science and Eng., 9(4) 24-31 (2007)

Cox, A., Computation in the Classroom: Open Source Physics Resources. American Physical Society, 2009 APS March Meeting (2009)

Christian, W., Building a Digital Library: Theory, Computation, and Education. American Physical Society, 2009 APS March Meeting (2009)

Cox, A., Computation in the Classroom: Open Source Physics Resources. American Physical Society, 2009 APS March Meeting (2009)

Christian, W. Interactive Computer-based Models and the Internet. American Physical Society, 75th Annual Meeting of the Southeastern Section of APS, Oct. 30-Nov. 1, 2008

Christian, W., M. Belloni y D. Brown, An opensource XML framework for authoring curricular materials. Computing in Sci. \& Eng., 8(5) 51-8 (2006).

Mzoughi, T., The Physic Source, the Digital Library for Teachers of Introductory Physics. AAPT 2010 Summer Meeting, Portland, Or, July 18, (2010).

\section{El Frente de Física}

Ezrailson, C.M., Mining The Physics Front for Teaching Resources. AAPT 2010 Summer Meeting, Portland, Or, Panel presentation BA04, July 18, (2010).

Ezrailson, C.M. How Using The Physics Front Digital Library Can Support Best Practices in Science. Meridian: A Middle School Computer Technologies Journal, 11(2) 1-4, (2008).

Ezrailson, C.M., The Physics Front: Resources for High School Physics \& Physical Science Teachers. 2007 AAS/AAPT Bulletin of the American Astronomical Society, 38 p 1067 (2007).

Ezrailson, C.M., Mining The Physics Front for Teaching Resources, AAPT 2010 Summer Meeting, Portland, Panel presentation BA04, July 18, (2010).
Lee, E.V., Physics To Go: an Outreach Digital Library. 2007 AAS/AAPT Joint Meeting, Bulletin of the American Astronomical Society, 38 p 1067 (2007).

Cole, R., S. Tooker, Physics to go: Web-based tutorials for CoLoS physics simulations. Frontiers in Education Conference, 6-9 Nov 1996.

\section{Adoptar-a-un-Físico}

Baldwin, K. The act "adopt a physicist" program. Physicist (Australian Institute of Physics), 37 (4) p 136, (2000).

Rand, K., y J. Clark, Adopt-a-Physicist: Connecting Physicists with High School Students. American Physical Society, APS March Meeting, March 5-9, abstract \#S21.002 (2007).

\section{Centro de Astronomía}

Brissenden, G. y S. Deustua, AstronomyCenter.org: Your Online Destination for Astronomy Education Resources. 2007 AAS/AAPT Bulletin of the American Astronomical Society, 38 p1067 (2007).

Gagne, M., P. Monahan, S. Deustua y B. Mason, Astronomy enter.org - A Digital Library for Astronomy Bulletin of the Am. Astronomical Soc. 37 p 373 (2004).

\section{Coalición del Profesor para la Educ. de la Física}

Finkelstein, N., C. Turpen, S. Pollock, M. Dubson, S. Iona, C. Keller y V. Otero, Evaluating a model of research-based practices for teacher preparation in a physics department: Colorado PhysTEC. Physics Educ. Research Conf., Salt Lake City-USA (2006)

Wells, L., R. Valenzuela, E. Brewe, L. Kramer, G. O'Brien y E. Zamalloa, Impact of the FIU PhysTEC reform of introductory physics labs. Physics Education Research Conf., Edmonton, AB, Canada (2008)

\section{El Intercambio del Cuántico}

Belloni, M., W. Christian y D. Brown, Open Source Physics Curricular Material for Quantum Mechanics, Computing in Science and Engineering, 9(4) p 2431, July/August (2007).

Belloni, M., W. Christian y B. Mason, Open Source and Open Access Resources for Quantum Physics Education. Spring 2008 CONFCHEM, 2008. 
Tabla 6. (continuación)

\section{Física para LLevar}

Hancock, L., SPS Internship: Working With Physics to Go. American Physical Society, 2008 Joint Fall Meeting of the Texas and Four Corners Sections of APS, AAPT, and Zones 13 and 16 of SPS, and the Societies of Hispanic and Black Physicists (2008)

Deml, A., A. 2006 SPS Summer Intern's Experiences, Reflections, and Future Ambitions. 2007 AAS/AAPT Bulletin of the American Astronomical Society, 38 p1094 (2006).

\section{El Núcleo}

Donnelly, D., Overview of the Student Collection on ComPADRE. 2007 AAS/AAPT Bulletin of the American Astronomical Society, 38 p 1067, (2007).

Donnelly, D., Overview of The Nucleus - The Student Collection of ComPADRE. AAPT 2010 Summer Meeting, Portland,Or-USA(2010).

Zaunbrecher, K.N., SPS Intern Contributions to ComPADRE and SOCK. 2007 AAS/AAPT Bulletin of the Am. Astronomical Soc., 38 p 1068 (2007).

\section{DISCUSIÓN Y CONCLUSIONES}

Una de las razones para la creación de ComPADRE fue la necesidad de mejorar los recursos existentes en el Centro de Recursos para Ciencias Físicas (PSRC) mediante el desarrollo de comunidades, el establecimiento de procedimientos para la presentación y archivo de contenidos, el establecimiento de un marco de metadatos para fácil acceso y la creación de objetos listo para usar como materiales para los cursos. También existía la percepción de que los instructores de la ciencia sólo utilizaban un número limitado de los recursos disponibles en su entorno inmediato, y que cuando realizaban búsquedas en el web de recursos adicionales, esto tomaba una cantidad insuperable de su tiempo para explorar y organizarlos.

Un aspecto muy importante de ComPADRE es la estructura técnica en la que se construyen los recursos de la comunidad. Construido con un diseño flexible, un miembro de una comunidad con las expectativas de un plan de estudios específico es capaz de obtener de la base de datos central las herramientas más adecuadas para una aplicación específica. Como Mason (2004) bien lo describe: "Por ejemplo, a pesar de que podrían utilizar la misma simulación de Java, un profesor de física de secundaria, y un profesor universitario enseñando un curso de nivel superior utilizan la simulación de diferentes maneras, la relacionan con diferentes tipos de actividades para los estudiantes, e incluso utilizan un lenguaje un poco diferente para describirlo." Además, no es raro que los estudiantes de Física tengan problemas para obtener una buena comprensión de los principios que se presentan en el aula. Esto es especialmente cierto cuando con aprendizaje de conceptos avanzados de la Mecánica Cuántica. Belloni, Christian y Brown (2007) muestran las ventajas de la utilización de materiales curriculares interactivos del proyecto Física de Código Abierto y señalan que " materiales curriculares cuidadosamente construidos, basados en computadoras y con simulaciones interactivas puede reducir la carga cognitivas de los alumnos."

El uso de tecnologías de la información como las que se presentan aquí pueden también generar colaboraciones académicas internacionales (Valderrama et al., 2011), sería necesario modificar y adaptar los programas educativos con el fin de dar a los estudiantes oportunidades para participar en aprendizaje electrónico (e-learning), lo que permitirá la utilización de recursos educativos desarrollados en varios países. Recursos educativos basados en la web, tales como los disponibles en la NSDL son herramientas muy poderosas que pueden ser utilizadas para promover la educación científica en todo el mundo. Específicamente, ComPADRE ha estado ejerciendo un papel importante en la formación de educadores de la ciencias, en el apoyo a los profesores de Física y en la ayuda a los estudiantes. También mediante el apoyo a un amplio espectro de instructores de las Ciencias Físicas, la asociación con los diseñadores curriculares y los investigadores educativos; proporcionando un lugar para una variedad de servicios en la web; e integrando un gran número de recursos de las sociedades profesionales. A pesar de que no se sabe hasta qué punto un sitio web como el que se describe aquí se utiliza, se espera que la información presentada en este documento servirá para crear un más amplio conocimiento en todo el mundo acerca de ComPADRE. Uno de los grandes atributos de este sitio web es que los educadores pueden encontrar en un solo lugar una enorme cantidad de recursos educativos que han pasado por un proceso de selección y que los usuarios pueden utilizar una gama de servicios bien desarrollados. Se recomienda a los lectores a explorar ComPADRE, compartir este artículo con los educadores de la Física, y también convertirse en miembros de la comunidad ComPADRE. 


\section{AGRADECIMIENTOS}

Este trabajo fue posible gracias al apoyo brindado por la Biblioteca de la Universidad de Northern Illinois University, DeKalb, II, EEUU. También se reconoce las contribuciones hechas por Adolfo Perilla, Lingüísta de la Universidad del Valle, Cali Colombia por la revisión del manuscrito y sus valiosos comentarios.

\section{REFERENCIAS}

Adams W.K. , S. Reid, R. LeMaster, S. B. McKagan, K. K. Perkins, M. Dubson y C. E. Wieman, $A$ Study of Educational Simulations Part I - Engagement and Learning, Journal of Interactive Learning Research, 19(3) 397-419 (2008).

Arms, W.Y. Digital Libraries, MIT Press, Cambridge, MA (2000).

Baki, A. y Ü. Çakıroğlu, Learning Objects in High School Mathematics Classrooms: Implementation and Evaluation, Computers \& Education, 55(4), 1459-1469 (2010).

Belloni, M., W.Christian, y D. Brown, Open Source Physics Curricular Material for Quantum Mechanics, Computing in Science and Engineering, 9(4), 24-31, July/August, (2007).

Cabral Vargas, B. Biblioteca Digital: Contenidos y Aprendizaje, Revista Interamericana de Bibliotecología, 29(2), 119-140 (2006).

Conceição, S.C.O., editor. Teaching Strategies in the Online Environment, Jossey-Bass, San Francisco, CA (2007).

Deustua, S., ComPADRE: Communities of Physics and Astronomy Digital Resources in Education, Mercury Magazine, 33(2), 19-23, March/April (2004). Disponible en línea en:

http://www.astrosociety.org/pubs/mercury/33_02/compadre.html (Consultado el 20 de Julio, 2011).

Esnault, L., editor. Web-based Education and Pedagogical Technologies: Solutions for Learning Applications, IGI Pub, Hershey, UK (2008).

Heckler, V., M.F. Oliveira Saraiva, y K.S. Oliveira Filho, Uso de Simuladores, Imagens e Animaç̃oes como Ferramentas Auxiliares no Ensino/Aprendizagem de Óptica, Revista Brasileira de Ensino de e Física, 29(2) 267-273 (2007).

Kay, R. y L. Knaack, Evaluating the Use of Learning Objects for Secondary School Science. Journal of Computers in Mathematics and Science Teaching, 26(4), 261-289 (2007).

McMartin, F., E. Iverson, A. Wolf, J. Morrill, G. Morgan y C. Manduca, The Use of Online Digital Resources and Educational Digital Libraries in Higher Education, International Journal on Digital Libraries, 9(1) 65-79, (2008).

Mason, B. ComPADRE: Resources for Physics and Astronomy Education. Project Kaleidoscope, Volume IV: What works, what matters, what lasts, 2004- present. Disponible en línea en: http://www.pkal.org/template2.cfm?c_id=1378 (Consultado el 20 de Julio, 2011), (2004).

Mason, B. Introducing ComPADRE, APS Forum on Education Newsletter, Fall, 19, (2007).

Morrison, G.R. et al. Designing Effective Instruction, 6th ed., Wiley, Hoboken, NJ (2011).

Osorio, N.L., The National Science Digital Library (NSDL): An Examination of its Role as a Digital Engineering Education Library. In Proceedings of the American Society for Engineering Education, Illinois-Indiana and North Central 2006 Conference. Technical Session B, Track 3, 10 p., in CDROM. Fort Wayne, IN: Indiana University - Purdue University Fort Wayne, (2006). 
Santos Miranda, M., A. Riposati Arantes y N. Studart, Objetos de Aprendizagem no Ensino de Física: Usando Simulações do PhET. XIX Simpósio Nacional de Ensino de Física - SNEF 2011 Manaus, AM, 30 de Janeiro a 04 de Fevereiro de 2011, 10 p. Disponible en línea en: http://www.sbf1.sbfisica.org.br/eventos/snef/xix/sys/resumos/T0137-1.pdf (Consultado el 20 de Julio, 2011) (2011).

Serrano, J.E. y P.S. Narváez, Uso de Software Libre para el Desarrollo de Contenidos Educativos, Formación Universitaria, 3(6), 41-50 (2010).

Studart, N. Objetos de Aprendizagem no Ensino de Física: Um Recurso Pedagógico Moderno para Professores e Alunos. En Práticas Pedagógicas, Linguagem e Mídias. Desafios à Pósgraduação em Educação em suas múltiplas dimensões. Helena Amaral da Fontoura and Marco Silva. Rio de Janeiro, Brazil: ANPEd Nacional, 2011, p 39-54. Disponible en línea en: http://www.fe.ufrj.br/anpedinha2011/ebook1.pdf\#page=39 (Consultado el 20 de Julio, 2011) (2011).

Tobias, S., y Duffy, T. M. , editors, Constructivist Theory Applied to Instruction: Success or Failure? Taylor \& Francis. New York, NY (2009).

Valderrama, J. O., Á. Sánchez y S. Urrejola, Colaboración Académica Internacional en Tecnologías de la Información y Docencia Virtual, Formación Universitaria, 2(6), 3-13 (2009).

Zia, L.L., The NSF National Science, Technology, Engineering, and Mathematics Education Digital Library (NSDL) Program. New Projects and a Progress Report. D-Lib Magazine, 6(10), 2001. Disponible en línea en: http://www.dlib.org/dlib/october00/zia/10zia.html (Consultado el 10 de Julio, 2011) (2001). 
